identified a significant association within the Class II MHC region.

Disclosure of Interest None Declared.

\section{OC-005 NOVEL PATHWAY-CENTRIC ANALYSIS REVEALS VARIANTS ASSOCIATED WITH TOXICITY AND RESPONSE TO THIOPURINES IN PATIENTS WITH INFLAMMATORY BOWEL DISEASE}

${ }^{1} \mathrm{PA}$ Blaker*, 'S Fong, ${ }^{2} \mathrm{~J}$ Walker, ${ }^{2} \mathrm{CM}$ Lewis, ${ }^{3} \mathrm{AM}$ Marinaki, ${ }^{1} \mathrm{JD}$ Sanderson, ${ }^{1} \mathrm{PM}$ Inving. ${ }^{1}$ Gastroenterology, Guys and St Thomas Hospitals' NHS Foundation Trust, London, UK; ${ }^{2}$ Genetics and Molecular Medicine, London, UK; ${ }^{3}$ Purine Research Laboratory, King's College, London, UK

\subsection{6/gutjnl-2014-307263.5}

Introduction Thiopurines remain the first line immunosuppressants recommended in the management of inflammatory bowel disease (IBD). Unfortunately, $30-40 \%$ of patients prescribed these agents develop adverse drug reactions or fail to derive therapeutic benefit. Candidate gene studies have identified loci that explain some of these treatment failures; however a substantial fraction of the genetic contribution remains undefined. Using whole thiopurine pathway analysis the aim of this study was to identify novel loci associated with toxicity and response to azathioprine (AZA)/mercaptopurine (MP) in patients with IBD.

Methods Genomic DNA was extracted from EDTA blood samples of 472 well-characterised IBD patients treated with AZA/ MP. We examined exome array data using the Illumina HumanExome Beadchip and restricted the analysis to variants associated with the thiopurine pathway as defined by the KEGG database (100 genes, 639 single nucleotide polymorphisms). Using a case-control design we firstly tested for genetic associations between patients with $(n=154)$ and without $(n=258)$ adverse drug reactions, and secondly for polymorphisms differentiating patients with $(n=188)$ and without $(n=141)$ response to thiopurines after 12 months of treatment. One year intervention-free clinical response was defined by 3 investigators (PB, PI, JS).

Results Following adjustment for principal components, the minor alleles at $A D K$ rs946185 ( $\mathrm{p}=0.0078$; OR 1.675), SLC28A1 rs2242046 ( $\mathrm{p}=0.0168 ;$ OR 1.600) and ABCA1 rs4149268 ( $\mathrm{p}=0.033$; OR 1.487) were associated with the development of drug toxicity, whereas the minor alleles at ABCB5 rs2301641 ( $\mathrm{p}=0.0170$; OR 0.608), ABCC4 rs4148549 $(\mathrm{p}=0.027$; OR 0.652) and AOX1 rs55754655 ( $\mathrm{p}=0.038$; OR 0.549 ) protected against it. The minor allele at RRM2 rs1130609 ( $\mathrm{p}=3.80 \times 10^{-5}$; OR 0.461), which codes a subunit of ribonucleotide reductase involved in the conversion of thioguanine nucleotide to deoxy-thioguanine nucleotide, and a higher normalised dose of AZA/MP were associated with protection from non-response. Conversely, the minor allele at ABCA1 rs2230808 ( $\mathrm{p}=0.008$; OR 2.585) and Crohn's disease ( $\mathrm{p}=$ $<0.001$; OR 5.007) were associated with non-response to treatment at 12 months.

Conclusion High-throughput sequencing using exome array technology has revealed new loci, other than thiopurine-S-methyltransferase, explaining toxicity and response to thiopurines. Validation of these markers in separate cohorts will allow the development of biomarker panels to predict outcomes prior to the start of treatment.

Disclosure of Interest None Declared.
OC-006 SAFETY AND EFFICACY OF LOW DOSE AZATHIOPRINE AND ALLOPURINOL CO-THERAPY: A LARGE SINGLE CENTRE EXPERIENCE

${ }^{1} \mathrm{P}$ Stamoulos*, ${ }^{1} \mathrm{E}$ Stournaras, ${ }^{1} \mathrm{C}$ Bull, ${ }^{1} \mathrm{M}$ Cowan, ${ }^{1} \mathrm{G}$ Mackenzie, ${ }^{1} \mathrm{~J}$ Stenner, ${ }^{2} \mathrm{~S}$ Coulthard, ${ }^{1}$ A Ansari. 'Gastroenterology, East Surrey Hospital, Redhill, UK; ${ }^{2}$ Institute of Cellular Medicine, Newcastle University Medical School, Newcastle, UK

\subsection{6/gutjnl-2014-307263.6}

Introduction The effectiveness of full dose azathioprine (FDA) for inflammatory bowel disease (IBD) has been questioned in recent scientific literature. A popular strategy to improve its outcomes recommends the use of low dose azathioprine with allopurinol co-therapy (LDAA) for patients profiled as "hypermethylators" (30\% of non-responders). The aim of this study was to determine the safety and efficacy of LDAA without using thiopurine metabolite (TM) profiling.

Methods Records of IBD patients treated with LDAA were retrospectively analysed.

Patients who had poor response and/or side-effects to FDA were offered LDAA by all Consultants whilst a single IBD physician also offered LDAA to thiopurine-naïve patients. Azathioprine dose was reduced to $25 \%$ of the thiopurine methyl transferase (TPMT) adjusted dose $(0.5 \mathrm{mg} / \mathrm{kg}$ for wild type and $0.25 \mathrm{mg} / \mathrm{kg}$ for heterozygotes) followed by conventional haematological monitoring. Non-adherence was assessed by TM measurements. Full response (FR) was defined as steroid free remission (Harvey Bradshaw index $\leq 3$, Truelove-Witts normal) for greater than 3 months after a 3 month induction period for LDAA.

Results Of 300 LDAA patients, adequate data was available for 295 cases. Group $1(\mathrm{G} 1)$ were treated $1^{\text {st }}$ line $(\mathrm{n}, 105)$ and Group 2 (G2) were switched from FDA to LDAA (n,190). Overall, for both groups, there were 207 (70\%) full responders (FR), 20 partial responders (PR) and 68 non-responders (NR). Full response rate was $78 \%$ in G1 and $66 \%$ in G2. The commonest indication for switching to LDAA was nonresponse to FDA (n,118).

Analysis of haematological indices revealed significant changes $(\mathrm{p}<0.05)$ in erythrocyte sedimentation rate, white cell count and platelet count after therapy induction.

Myelotoxicity occurred in 5 patients (all NR, WCC >2 and $<3.5$ ) and 12 patients had asymptomatic hepatotoxicity (ALT range: 100-700) which resolved by increasing allopurinol to $200 \mathrm{mg}$ in 9 patients (all FR).

Time on treatment: 208 patients took LDAA for more than twelve months with a median length of therapy of 24 months.

Conclusion Appropriately dosed LDAA therapy delivers a therapeutically effective dose of azathioprine without the

\begin{tabular}{lllll} 
Abstract OC-006 Table 1 & \multicolumn{5}{c}{ Clinical response (n) } \\
\hline Indication & FR & PR & NR & Total \\
\hline AZA/6MP Naive (LDAA without FDA exposure) & 82 & 7 & 16 & 105 \\
Switched from FDA to LDAA & 125 & 13 & 52 & 190 \\
Poor response & 81 & 10 & 27 & \\
GI intolerance & 16 & $n / a$ & 11 & \\
Hepatotoxicity & 7 & 1 & 3 & \\
Myelotoxicity & 13 & 2 & 1 & \\
"Flu-like" symptoms & 3 & n/a & 1 & \\
Other & 5 & n/a & 9 & \\
\hline
\end{tabular}


need for dose escalation. It appears to be more effective, better tolerated and safer (less haematological disturbance) than FDA. These results will serve to allay the fear of toxicity of LDAA and question the need for thiopurine metabolite level profiling prior to using this apparently superior therapeutic approach.

Disclosure of Interest None Declared.

\section{OC-007 HAEMOPOETIC STEM CELL TRANSPLANTATION FOR SEVERE RESISTANT CROHN'S DISEASE: PRELIMINARY EVIDENCE FOR DURABLE BENEFIT}

C Hawkey* on behalf of The ASTIC Trialists (listed at http://www.nottingham.ac.uk/ research/groups/giandliverdiseases/nddc-clinical-trials/astic-trial/centres-and-members.asp). Nottingham Digestive Diseases Centre, University of Nottingham, Nottingham, UK

\subsection{6/gutjnl-2014-307263.7}

Introduction The Autologous Stem Cell Transplantation International Crohn's Disease (ASTIC) Trial shows haemopoetic stem cell transplantation (HSCT) to be effective over one year in Crohn's disease, but its durability remains to be established.

Methods ASTIC is a multicentre parallel group randomised controlled trial in patients with impaired quality of life due to Crohn's Disease that is resistant to established treatments. All patients undergo stem cell mobilisation before HSCT given immediately (one month: early HSCT) or after a delay of thirteen months (late HSCT). This abstracts describes currently available data over 2 years and includes the first report of changes occurring in the first year transplantation in the late HSCT group.

Results A full analysis of progress over one year has shown a reduction in Crohn's Disease Activity Index (CDAI) from 326 (range 163-512) to $162(12-506)$ and in the SES-CD endoscopic score from $13(5-33)$ to $3(0-200)$ in patients following early HSCT $(\mathrm{n}=23)$ compared to 354 (91-581) to 298 (70-589, active vs control $p=0.01)$ and $13.5(0-36)$ to $7(1-27$, active vs control $\mathrm{p}=0.02)$ respectively in the control group prior to transplantation $(\mathrm{n}=22)$. The Table shows data for those patients (approximately 50\%) with full data currently available to two years (one year after transplantation in the control group).

Scores for the IBD-Q quality of life index improved from $123(103-144)$ to 165 (125-206) following early HSCT and were maintained at 157 (126-213). Scores rose from 108 (79$136)$ to 147 (108-188) in the year following delayed HSCT.

Conclusion If full data (available June 2014) confirm these preliminary results, it would support the notion that improvements in CDAI, endoscopic appearances and quality of life benefit persist and may possibly increase over the second year following transplantation. One year data in the delayed group show a similar magnitude of effect to that seen in patients undergoing early transplantation.

Disclosure of Interest None Declared.

\section{Endoscopy section free papers}

\section{OC-008 ENDOSCOPIC SUBMUCOSAL DISSECTION CAN TRANSFORM THE MANAGEMENT OF PATIENTS WITH UPPER GASTROINTESTINAL SUBMUCOSAL TUMOURS: RESULTS FROM A UK SERIES}

R Bhattacharyya*, P Bhandari on behalf of Portsmouth research group. Gastroenterology, Portsmouth Hospitals NHS Trust, Cosham, Portsmouth, UK

\subsection{6/gutjnl-2014-307263.8}

Introduction It is very difficult to establish an accurate diagnosis for upper GI submucosal tumours. Biopsy during endoscopy cannot go deep enough. EUS is unable to give a tissue diagnosis. The risks of surgical resection are higher than the benefits as the lesion may very well be benign. As a result most of these patients keep having endoscopic surveillance as 'possible' GISTs.

Methods A retrospective cohort study of patients undergoing ESD for upper GI submucosal tumours. They were all referred to us as possible GISTs that were found to be growing in size on surveillance. ESD was carried out in all these cases. As these lesions are mostly bulky, gravity and patient positioning were utilised as traction during ESD to achieve deroofing and enucleation of these tumours. Any complications were recorded. Endoscopic follow up was performed to assess for incomplete resection or recurrence.

Results 21 submucosal lesions were resected by ESD between 2007 and 2013. 7 were oesophageal, 10 gastric and 4 duodenal. Sizes ranged from 10 to $35 \mathrm{~mm}$. Endoscopic clearance was achieved in all cases. Histology showed a wide range of diagnoses, mostly benign (table). There was 1 complication; a microperforation which was identified and clipped intraprocedurally, giving a complication rate of $4.7 \%$. On follow up, there was 1 recurrence (recurrence rate 4.7\%) which was managed

\begin{tabular}{|c|c|}
\hline Diagnosis & Number \\
\hline Granular cell tumour & 3 \\
\hline GIST & 2 \\
\hline Leiomyoma & 2 \\
\hline Pancreatic acinar tissue & 1 \\
\hline Carcinoid & 6 \\
\hline Lipoma & 2 \\
\hline Inflammatory fibroid polyp, hyperplastic polyp & $1+1$ \\
\hline Synovial sarcoma & 1 \\
\hline Gangliocytic paraganglioma & 1 \\
\hline
\end{tabular}

\begin{tabular}{|c|c|c|c|c|c|c|}
\hline & \multicolumn{2}{|c|}{$\operatorname{CDAI}(n=12$ and 14$)$} & \multicolumn{2}{|c|}{ SES-CD ( $n=13$ and 13$)$} & \multicolumn{2}{|c|}{ EQ5D VAS $(n=9$ and 10$)$} \\
\hline & Early HSCT & Late HSCT & Early HSCT & Late HSCT & Early HSCT & Late HSCT \\
\hline Baseline & $338(264-473)$ & $354(264-473)$ & $13(8.5-25)$ & $14(4.5-19.5)$ & $56(39-65)$ & $35(29-60)$ \\
\hline 1 year & $162(73-280)^{*}$ & $288(209-368)$ & $3(1.5-10)^{*}$ & $6(3-20)$ & $80(68-88)^{*}$ & $50(21-70)$ \\
\hline 2 year & $90(36-231)^{*}$ & $155(84-300)^{*}$ & $3(0-10.5)^{*}$ & $2(0-6.5)^{*}$ & $87(64-91)^{*}$ & $74(43-83)^{*}$ \\
\hline
\end{tabular}

